\title{
5 The problem of probabilities
}

A second issue that the argument from the Basic Representation Theorem raised, besides the problem of intertheoretic comparisons, was the question of how to explicate probabilities. In the Basic Representation Theorem, the concept of a probability distribution over axiologies figured as a primitive. The prospects over which the m-value relation ranges were defined as leading to particular outcomes with particular probabilities, while different axiologies have particular probabilities of being true. So the concept of a probability distribution over axiologies appeared as a primitive in the very definition of these prospects. That was useful to focus on the other problems I've addressed. And it follows a trend in recent decision theory, where some authors have advocated taking credences as a primitive. ${ }^{105}$ However, at least in the theory of axiological uncertainty, this primitivism seems ultimately unsatisfying. Suppose you say the human-welfare-theory has a probability of 0.05 . Unless you give some account of what you mean by that, we arguably don't understand your assertion-or the difference between your statement and the claim that the probability is 0.1 , or 0.01 . That's particularly so because the notion of an axiological probability distribution is highly complex. It's triply quantitative: specifying quantitative probabilities attached to intratheoretically cardinal axiologies among which cardinally significant comparisons hold. The last point is especially important. It's not enough to know what it is for a particular axiological ordering to have considerable weight in your m-value facts, or even to be in some sense three times as weighty as another ordering. We also need to know what it is for such an axiology to have that weight in virtue of a high probability, but a relatively deflated value function, rather than in virtue of an inflated value function, but a relatively low probability. It's not plausible that our intuitive understanding of such a rich notion goes very far. So we need an explication of what we mean by axiological probabilities. That's the task of the present chapter.

The question of how to explicate probabilities is connected with a larger question about how to understand EVM and its normativity, which I've briefly indicated (on pages 23 and 60), but which requires further clarification. 'Axiological probabilities' could mean two different things. It could mean subjective probabilities-the actual credences you have in particular versions of axiological orderings. Or it could mean evidential probabilities-the credences your evidence warrants in particular versions of axiological orderings. Accordingly, the notion of ' $m$-value' and the core claim of EVM could be understood more, or less, subjectively. On a more subjective interpretation of $\mathrm{m}$-value, a prospect is $\mathrm{m}$-better than

\footnotetext{
105 See e. g. Eriksson and Hájek (2007).
}

Ә Open Access. (c) 2021 Stefan Riedener, published by De Gruyter. (cc))BY-NC-ND This work is licensed under the Creative Commons Attribution-NonCommercial-NoDerivatives 4.0 International License. 
another if it's better in light of your subjective credences in particular versions of axiological orderings-or in light of your credences in axiological orderings and the intertheoretic comparisons you make between them. (Again, we can interpret credences in axiological orderings plus specific comparisons between them simply as credences in specific versions of these orderings.) On this interpretation, EVM figures as a rational coherence constraint on your m-value judgments-your judgments about which prospects are m-better than which. It says that however precisely you're axiologically uncertain, you must assign certain probabilities to your axiologies, compare them in a certain way, and evaluate prospects in terms of their expectation relative to your credences and comparisons. If you do, your m-value judgments are (subjectively) correct. I'll call this Subjectivist EVM.

Let me clarify. I suggested in the last chapter (Section 4.1) that subjectivism can't be the whole story. Unless there's some norms about which credences and comparisons are more reasonable than which, as long as you satisfy our coherence constraints, we'll have no grounds at all to criticise your m-value judgments. This seems unduly permissive. Intuitively, m-value judgments on which it's always m-best for you to act as an Übermensch and do what you please seem in some sense worse than others (even if they satisfy our constraints). More fundamentally, unless there are such norms, we arguably can't speak of 'credences' in the first place. But constructivism provides a standard of reasonableness for credences and comparisons. In light of it, we can speak of credences, and criticise some of them as epistemically flawed. So given constructivism, a focus on subjective credences and comparisons seems viable. Subjectivist EVM doesn't deny that there are objective grounds for criticising your credences in particular versions of axiological orderings (even beyond coherence). Perhaps in light of Conservatism and Coherence, it's inappropriate for you to believe in such a massively inflated version of the Nietzschean axiology. Subjectivist EVM just isn't interested in whether your credences and comparisons are reasonable in this sense. Instead, it's interested in whether you evaluate your prospects in a subjectively reasonable manner, given your credences and comparisons. We might say it's analogous to a subjective version of decision theory. This theory doesn't deny that there are grounds for criticising your preferences and non-normative credences (even beyond coherence). It's not thoroughly Humean. Perhaps believing that the world will be destroyed in the coming ten years means a gross insensitivity to our evidence. Perhaps preferring the destruction of the world to the scratching of your finger means an overestimation of your itch. Subjective decision theory doesn't ask whether your beliefs and preferences are reasonable in this sense. It just asks which prospects you should, subjectively, prefer to which given your credences and preferences. What I'll call Subjectivist EVM does the same. It's interested in a thoroughly subjective 'ought', not denying there's a more objective one too. Thus 
it says that if you evaluate prospects in terms of their expectation relative to your credences and comparisons, your m-value judgments are (subjectively) correct. If we're interested in such Subjectivist EVM, we need to know what it is for you to have a certain subjective credence in a particular theory.

On a more objective interpretation of $\mathrm{m}$-value, a prospect is $\mathrm{m}$-better than another if it's better in light of the credences that your evidence warrants, and the intertheoretic comparisons that are correct (in a constructivist manner, in light of the relevant empistemic norms). On this interpretation, EVM figures as a more substantive theory of $\mathrm{m}$-value. It says that axiologies have certain evidential probabilities and compare in a certain way, and that a prospect is $\mathrm{m}$-better than another if and only if it has the greater expected moral value relative to these probabilities and comparisons. So even if you evaluate prospects in terms of their expectation relative to your credences and comparisons, your m-value judgments might be false. I'll call this Objectivist EVM. It's analogous to a thoroughly objective version of decision theory, which doesn't just ask which prospects you should prefer to which given your credences and concerns-but also which credences and concerns you should have in the first place. If we're interested in Objectivist EVM, we need to know what it is for your evidence to warrant a certain credence in a particular theory.

Both Subjectivist and Objectivist EVM are interesting. There are reasons to explore the objectivist theory. It's often more in line with our actual practice. Often when we reflect on what's (m-)best in the face of arguments for competing axiologies, we try to make judgments that are sensitive to our evidence and the correct comparisons. We don't try to make judgments that are sensitive to the credences and comparisons we happen to find ourselves with. Metaphorically speaking, our credences are transparent in our judgments: we don't look at them, but with them or through them in judging our options. ${ }^{106}$ Moreover, Objectivist EVM is more thoroughly normative. If you satisfy Subjectivist EVM, but have wildly inappropriate axiological credences, your m-value judgments are still importantly suboptimal. But there are reasons to explore the subjectivist theory too. Subjectivist EVM doesn't seem alien to our practice. We can certainly try to make judgments that are sensitive to the credences and comparisons we happen to have, or that we settled upon after first reflecting on our evidence. It's not that we necessarily try to reach objectivist verdicts. More positively, there's a sense in which Subjectivist EVM focuses most clearly on the normativity of the theory of axiological uncertainty. Suppose you maximise expected value relative to your credences and comparisons, but have inappropriate credences. Then you aren't making a

106 I thank Felix Koch for pointing this out to me. 
mistake about whether EVM, or My Favourite Theory, or some meta-deontological theory of axiological uncertainty is true, say. You're making an epistemic mistake. In this sense, as long as you're satisfying Subjectivist EVM, you're not making a mistake peculiar to the theory of axiological uncertainty. Finally, the notion of a subjective probability or credence is arguably conceptually more fundamental than that of an evidential probability or evidentially warranted credence. Only if we know what it is to have certain credences can we know what it is for certain credences to be warranted.

Fortunately, the two theories raise similar questions, and can be developed in similar fashions. So our focus doesn't matter very much. I'll generally start by elaborating on Subjectivist EVM, and then translate my arguments into Objectivist EVM. In line with my approach so far, I'll introduce an explication of axiological probabilities on the basis of a representation theorem (Section 5.1). I'll outline how we can apply a theory based on this explication in our practical decisionmaking (Section 5.2). And I'll then defend the explication against objections. I argue that representation theorems provide our best account of axiological probabilities, and outline the implications of this claim for the normativity of EVM (Section 5.3). Since all of this is controversial territory, I'll end the chapter by introducing Weighted Value Maximisation-a theory of axiological uncertainty that eschews the notion of credences altogether (Section 5.4).

\subsection{The Representation Theorem for Probabilities}

There are various possible answers to our question about what you mean by saying your credence in the human-welfare-theory is 0.05. But not all answers are equally good. Consider the

Simple Explication: That your credence in $T_{i}$ is $p_{i}$ means that when you consider the set of possible axiologies, consider how confident you feel about each axiology in light of the evidence for and against it, and try to associate a nonnegative number to each of them, such that the numbers add up to 1 and reflect your confidence, you associate with $T_{i}$ the number $p_{i}$.

This might explain what you mean by saying your credence in the human-welfaretheory is 0.05 . You might mean you distributed numbers to axiologies in accordance with your feelings of confidence and the human-welfare-theory ended up getting 0.05. However, this isn't a very good explication. For one thing, the Simple Explication doesn't seem to pick out something we'd intuitively identify as your degrees of belief. Suppose for some reason you associate the number zero to the human-welfare-theory on introspection. If in your actions and attitudes, and in 
your judgments about which prospects are m-best, you give considerable weight to that theory, then intuitively you do have credence in it. You simply misjudged your confidence. We need to pick out something closer to our intuitive concept of credences. For another thing, the Simple Explication doesn't seem to pick out something normatively very relevant. We can perform such an exercise of introspection. But assigning numbers to axiologies will soon seem a rather arbitrary stab in the dark. It would seem reckless to ground the most important decisions in our life solely on this intuitive list of numbers. We need to pick out something normatively more important.

A prominent candidate route is again via representation theorems. Let's start with some background. One of the first to explicate credences was Bruno de Finetti $(1980,62)$. He suggested that your credence in a proposition $p$ is the rate $x$ for which you'd be indifferent between receiving any sum of money $S$ if $p$ is true, and receiving $x S$ with certainty. So for you to have a credence of 0.25 that it will rain today, say, is for you to be indifferent between receiving $€ 1$ if it rains and the certain gain of $€ 0.25$. The obvious problem with this is that it seems to misrepresent your beliefs if money has diminishing marginal value for you. If you prefer a sure gain of $£ 1$ million to the bet that gives you $£ 2$ millions if and only if some coin lands tails, you needn't think the coin isn't fair. You might simply care more about the first than the second million. ${ }^{107}$ For this reason, the account given by Frank Ramsey (1990) was more promising. Ramsey provided a simple theorem showing that if your preferences satisfy certain conditions you can be represented as maximising expected utility with respect to relevantly unique probability and utility functions-which needn't conform linearly to any other independently specifiable quantity. According to Ramsey, this probability function can be interpreted as representing your credences. Ramsey's account was further refined by Leondard Savage (1954), who provided a conceptually more sophisticated theorem. The main tenet of all these explications is that to have a particular credence in a proposition is to give that proposition a particular weight in your preferences under uncertainty. Representation theorems show that if your preferences satisfy certain conditions, you maximise expected utility with respect to certain utility and probability functions. And according to the explications, we can then treat the probability function as specifying your actual credences.

I think this idea is promising. However, if we're to explain your axiological credences, we can't simply focus on your preferences. Perhaps you just don't care about moral value. Or perhaps you also care about many things besides it-your

107 See e. g. Eriksson and Hájek (2007) and Hájek (2019) for other famous objections against De Finetti's interpretation. 
self-interest, the welfare of your family, deontological constraints, or whatever. So you may give little weight in your preferences to the human-welfare-theory, say, and still find it plausible as an axiology. We need to separate your axiological and non-axiological concerns. We can do so by focusing on which prospects you find m-better than which. In other words, instead of explicating credences in terms of preferences, we can do so in terms of $m$-value judgments. Your credence $p_{i}$ in an axiology $T_{i}$ is the weight you give that axiology in your m-value judgments. I'll refer to this as the judgment-explication of axiological credences. So what I'll look for is a set of conditions $\mathcal{C}$ such that, if your m-value judgments satisfy $\mathcal{C}$, you maximise expected utility relative to a unique probability and a relevantly unique utility function. And I'll then assume that the probability function represents your axiological credences. Again, I treat this as an explication. The claim isn't that our theorem will identify the credences you really have. There's no such thing. Rather, the probabilities from this theorem pick out something close to our intuitive concept of credences, and something normatively important. That will be my claim in what follows.

\section{Axiological Probabilities}

So far, I've spoken rather impersonally about what 'our axiologies' and 'the mvalue facts' are. But according to Subjectivist EVM, you should evaluate your prospects relative to your credences and values. So we now need to focus on the axiologies you consider, and the $\mathrm{m}$-value facts relative to the credences you have in them. We need to reinterpret our formal framework slightly. To determine what your axiologies are, I'll assume you make (considered) judgments about prospects in $\mathcal{O}$, of the form 'according to axiology $T_{i}, a$ is better than $b$ '. I represent your judgments of this kind by 'your $\succeq_{i}$ ', to indicate that the set of these axiologies is the set you are considering. From these judgments, we can read off what axiological orderings you're considering. For instance, if you judge that according to $T_{1}$, a prospect is better than another if and only if it expectably leads to more human welfare, then, we'll assume, by $T_{1}$ you mean the human-welfare-theory.

However, we need to know not only what orderings, but also what versions of these orderings you're considering-or how you're comparing your orderings against each another. To that end, I'll assume you make (considered) m-value judgments about prospects in $\mathcal{Q}$. We might think of these as counterfactual judgments of the form 'if the probability distribution over axiologies was $P, a$ would be m-better than $b$ '. I represent these judgments by 'your $\geq_{m}$ ', to indicate that these are what you take to be the counterfactual m-value facts. From these judgments, we can read off how you're comparing your axiologies against each other. Suppose 
you consider the human-welfare-theory $T_{1}$ and the non-human-animal-welfaretheory $T_{2}$. And you judge that if they had a probability of $1 / 3$ and $2 / 3$ respectively, it would be equally $\mathrm{m}$-bad to inflict a given amount of pain on a human or on a non-human animal. Then, we'll assume, you think the value of a given amount of human welfare, according to $T_{1}$, is twice as great as that of the same amount of animal welfare, according to $T_{2}$.

However, we now also need to know what credence you have in these theories. To that end, we need a third set of prospects, beyond $\mathcal{O}$ and $\mathcal{Q}$. More specifically, we need a set $\mathcal{K}$ of probability distributions on $X$, in which the probability distribution over axiologies isn't specified. Intuitively, a prospect in $\mathcal{K}$ may be, say, that a person will suffer if and only if $T_{1}$ is true, or that a person will suffer if and only if $T_{1}$ is true and a non-human animal if and only if $T_{2}$ is true. I'll use cursive letters $a, b, c \ldots$ to refer to prospects in $\mathcal{K}$. Formally, these prospects can be represented as the set of theory-dependent probability distributions on $X-$ i. e., $\mathcal{K}=\left\{a: I \times X \rightarrow \mathbb{R}_{+} \mid \sum_{x \in X} a(i, x)=1 \forall i \in I\right\}$. The second just-mentioned prospect, say-on which a person will suffer if and only if $T_{1}$ is true and a non-human animal if and only if $T_{2}$ is true-will thus be represented as the function $a$ in $\mathcal{K}$ that assigns 1 to the pair of $T_{1}$ and the fact that the person suffers, 1 to the pair of $T_{2}$ and the fact that the non-human animal suffers, and 1 to the pairs of all other theories and the fact that nothing happens. Note that in a prospect in $\mathcal{K}$, the numbers assigned to outcomes sum to 1 within each axiology, not across axiologies. So a prospect in $\mathcal{K}$ doesn't specify an underlying probability distribution over axiologies. I'll assume you also make (considered) $\mathrm{m}$-value judgments about prospects in $\mathcal{K}$. We might think of these as non-counterfactual judgments of the form ' $a$ is m-better than $b$ ', relative to your credences. I represent these judgments by 'your $\widetilde{\succeq}_{m}$,' (or 'your $\widetilde{\succ}_{m}$ ' and 'your $\tilde{\sim}_{m}$ '), to indicate that these are what you take to be the $\mathrm{m}$-value facts relative to your credences. So I use a tilde to distinguish them from your judgments $\succeq_{m}$ on $\mathcal{Q}$.

If we know your judgments about $\mathcal{Q}$, then from your judgments about $\mathcal{K}$, we can read off what credences you have in your axiologies. By considering your counterfactual judgments ('If the probability distribution over theories was $P, a$ would be m-better...'), we can detect your values. The function representing these judgments is a pure reflection of values, since the probabilities are already given in the prospects. And knowing your values, we can then detect your probability distribution by considering your ordinary judgments ('Actually, $a$ is m-worse...'). Suppose, as we assumed above, that in your m-value judgments about $\mathcal{Q}$, you judge that if $T_{1}$ and $T_{2}$ had a probability of $1 / 3$ and $2 / 3$ respectively, it would be equally $\mathrm{m}$-bad to inflict a given amount of pain on a human or on a non-human animal. So you think the value of a given amount of human welfare, according to $T_{1}$, is twice as great as that of the same amount of animal welfare, according to $T_{2}$. 
And suppose that in your m-value judgments about $\mathcal{K}$, you judge that actually it's equally m-bad to inflict a given amount of pain on a human or twice that amount on a non-human animal. Then, if $T_{1}$ and $T_{2}$ are the only axiologies you consider, we'll assume you actually have a credence of $1 / 2$ in both of them.

That's the rough idea. To sum up, we'll need three kinds of judgments from you in order to get a relevant expected utility representation: judgments about which prospects are better than which according to your axiologies (represented by your $\succeq_{i}$ on $\mathcal{O}$ ), judgments about which prospects would be m-better than which given that axiologies had certain probabilities (represented by your $\succeq_{m}$ on $\mathcal{Q}$ ) and judgments about which prospects are m-better than which relative to your credences (represented by your $\tilde{\succeq}_{m}$ on $\mathcal{K}$ ).

Three notes about this framework are in order. First, the set $\mathcal{Q}$ still presupposes that we understand what it means that a particular axiology has a particular probability. One may worry that we don't-indeed, that it's precisely because we don't understand this that we needed a new framework, and that this framework thus mustn't feature our set $\mathcal{Q}$. But I think we can answer this worry. What I'd doubt is a pretheoretic understanding of quantitative subjective or evidential probabilities. The relevant notion in $\mathcal{Q}$ can be understood differently. For example, we might imagine that God determined the true axiology on the basis of a device involving an unpredictable quantum mechanical phenomenon, and understand the prospects in $\mathcal{Q}$ in terms of objective propensities of that device. We can assume you satisfy Lewis' (1980) Principal Principle, as part of our understanding of subjective probabilities. And given this assumption, we can take your m-value judgments about $\mathcal{Q}$ to be a pure reflection of your values. Judgments like 'If the probability distribution over theories was $P$, ...' could be understood as meaning: 'Supposing God's device had propensities represented by $P$, ...'. This adds another complexity to my framework. But I think it's only that: an extra complexity. The presupposition of $\mathcal{Q}$ doesn't make our explications circular, incoherent, or overly primitivist.

Second, note that the sets $\mathcal{K}$ and $\mathcal{Q}$ both specify an underlying probability distribution over outcomes (relative to each axiology). In this sense, I'm still taking purely descriptive probabilities as given primitives. In principle, this is problematic, for similar reasons as it was problematic to take axiological credences as a primitive. However, my main concern is with axiological uncertainty. This problem is complicated enough. To keep it as simple as possible, I'll assume purely descriptive probabilities as primitives throughout.

Third, the set $\mathcal{K}$ includes all theory-dependent probability distributions over outcomes. In particular, and as illustrated by the above example, it includes prospects that lead to different (probability distributions over) outcomes, depending on which axiology is true. That is, for some $a$ in $\mathcal{K}$, some $x$ in $X$, and some 
$T_{i}$ and $T_{j}, a(i, x) \neq a(j, x)$. As with the analogous prospects in $\mathcal{Q}$, these prospects won't represent any natural, practical options. There's no natural act which leads to a non-human animal suffering if one theory is true, and a person suffering if another theory is true. But as with the prospects in $\mathcal{Q}$, such prospects seem conceptually possible (see page 23). So I think it isn't a problem for our framework that $\mathcal{K}$ includes them. On the contrary, it would be a drawback if it didn't. Apart from this somewhat non-practical aspect, judgments about $\mathcal{K}$ are fairly natural. Indeed, in some sense they're more straightforward than judgments about $\mathcal{Q}$. They don't require us to counterfactually imagine a specific probability distribution over axiologies. They just require us to judge how we'd evaluate prospects in light of our actual uncertainty or evidence. So I take it it's plausible to assume that we can make considered $m$-value judgments about $\mathcal{K}$ (as well as about $\mathcal{O}$ and $\mathcal{Q}$ ).

Given this framework, we can state our new theorem. It will again feature the three conditions of the Basic Representation Theorem: that all your $\succeq_{i}$ and your $\succeq_{m}$ are vNM-conformable, and that your $\succeq_{m}$ satisfies the Pareto Condition with respect to your $\geq_{i}$. As we know from the Basic Representation Theorem, if your judgments satisfy these conditions, there's a function $u: I \times X \rightarrow \mathbb{R}$, unique up to positive affine transformation, such that for all $\boldsymbol{a}$ and $\boldsymbol{b}$ in $\mathcal{Q}$, all $a$ and $b$ in $\mathcal{O}$ and all $i$ in $I$,

$$
\begin{gathered}
\boldsymbol{a} \geq_{m} \boldsymbol{b} \text { iff } \sum_{i \in I, x \in X} \boldsymbol{a}(i, x) u(i, x) \geq \sum_{i \in I, x \in X} \boldsymbol{b}(i, x) u(i, x), \quad \text { and } \\
a \geq_{i} b \text { iff } \sum_{x \in X} a(x) u(i, x) \geq \sum_{x \in X} b(x) u(i, x) .
\end{gathered}
$$

We now need to relate this to your judgments about $\mathcal{K}$. To that end, we need two new conditions. The first is that your $\widetilde{z}_{m}$ on $\mathcal{K}$ also satisfies the von NeumannMorgenstern axioms. Define $p a+(1-p) b$ in $\mathcal{K}$ as the prospect that leads to $a$ with probability $p$, and to 6 with probability $(1-p)$, hence $(p a+(1-p) b)(i, x)=p a(i, x)+$ $(1-p) b(i, x)$ for all $(i, x)$ in $I \times X$. For a reflexive binary relation $\tilde{z}$ on $\mathcal{K}$, our axioms are

Transitivity $\mathcal{K}_{\mathcal{K}}$ for all $a, b$ and $c$ in $\mathcal{K}$, if $a \tilde{\geq} b$ and $b \tilde{\succeq} c$, then $a \tilde{\geq} c$;

Completeness $_{\mathcal{K}}$ : for all $a$ and $b$ in $\mathcal{K}, a \tilde{z} b$ or $b \tilde{z} a$;

Independence $_{\mathcal{K}}$ : for all $a, b$ and $c$ in $\mathcal{K}$ and $\left.p \in\right] 0,1[$, if $a \tilde{>} b$, then $p a+(1-$ $p) c \tilde{>} p \sigma+(1-p) c$; and

Continuity $_{\mathcal{K}}$ : for all $a, b$ and $c$ in $\mathcal{K}$, if $a \tilde{>} b$ and $b \tilde{>} c$, then there exist $\left.p, q \in\right] 0,1$, such that $p a+(1-p) c \tilde{r} b$ and $b \tilde{>} q a+(1-q) c$.

If a reflexive relation $\tilde{z}$ on $\mathcal{K}$ satisfies these conditions, I'll again say it's 'vNMconformable'. For two functions $u: I \times X \rightarrow \mathbb{R}$ and $v: I \times X \rightarrow \mathbb{R}$, say that $v$ is a positive unit-comparable transformation of $u$ if there are $s$ and $t_{i} \in \mathbb{R}, s>0$, 
such that $v(i, x)=s u(i, x)+t_{i}$ for all $i$ in $I$ and $x$ in $X$. It can be shown ${ }^{108}$ that if $\tilde{\succeq}_{m}$ is vNM-conformable, there's a function $v: I \times X \rightarrow \mathbb{R}$, unique up to positive unit-comparable transformation, such that for all $a$ and 6 in $\mathcal{K}$,

$$
a \tilde{\succeq}_{m} b \text { iff } \sum_{i \in I, x \in X} a(i, x) v(i, x) \geq \sum_{i \in I, x \in X} b(i, x) v(i, x) .
$$

However, this isn't enough for our purposes. For all we know, $v$ might be a random utility function without any relation to your axiologies or your m-value judgments about $\mathcal{Q}$. We need to ensure that the value functions $v$ from (5.3) and $u$ from (5.1) and (5.2) are related to each other-or more precisely, that the function $v$ can be understood as a product of your values, as specified by $u$, and your credences, as specified by a probability function $P$ on $I$.

So we need a further substantive condition. We need to assume that $\succeq_{m}$ and $\tilde{\succeq}_{m}$ are value-consistent-so that the differences between them can be fully explained by the underlying probabilities in $\mathcal{Q}$. To state this condition, let $\mathcal{Q}^{+} \subset \mathcal{Q}$ be the set of prospects in $\mathcal{Q}$ on which all axiologies have a positive probability, i. e. $\mathcal{Q}^{+}=$ $\left\{\boldsymbol{a} \in \mathcal{Q} \mid \sum_{x \in X} \boldsymbol{a}(i, x)>0 \quad \forall i \in I\right\}$. Define a function $L: \mathcal{Q}^{+} \rightarrow \mathcal{K} ; \boldsymbol{a} \mapsto L(\boldsymbol{a})$, such that for all $i$ in $I$ and $x$ in $X$,

$$
L(\boldsymbol{a})(i, x)=\boldsymbol{a}(i, x) / \sum_{y \in X} \boldsymbol{a}(i, y) .
$$

Intuitively, $L$ turns a prospect in $\mathcal{Q}^{+}$into the corresponding prospect in $\mathcal{K}$ in which the underlying probabilities have been scraped out. For some $i$ in $I$ and $a$ and $b$ in $\mathcal{K}$, say that $a$ and 6 agree outside $i$ if for all $j$ in $I$ with $j \neq i$, and all $x$ in $X$, $a(j, x)=b(j, x)$; and similarly for some $\boldsymbol{a}$ and $\boldsymbol{b}$ in $\mathcal{Q}$. For some $i$ in $I$ and binary relations $\succeq$ on $\mathcal{Q}$ and $\check{z}$ on $\mathcal{K}$, say that $i$ is null if $(i)$ for all $a$ and $b$ in $\mathcal{K}$ that agree outside $i, a \approx b$, and (ii) there exist $\boldsymbol{a}$ and $\boldsymbol{b}$ in $\mathcal{Q}$ that agree outside $i$ such that $\boldsymbol{a}>\boldsymbol{b}$. Say that $i$ is non-null if there are $a$ and $b$ in $\mathcal{K}$ that agree outside $i$ such that $a \tilde{\succ} 6$. Now for binary relations $\succeq$ on $\mathcal{Q}$ and $\check{\succeq}$ on $\mathcal{K}$, define the

Consistency Axiom: For all $i$ in $I$ and all $\boldsymbol{a}$ and $\boldsymbol{b}$ in $\mathcal{Q}^{+}$, if $\boldsymbol{a}$ and $\boldsymbol{b}$ agree outside $i$ and $L(\boldsymbol{a}) \tilde{>} L(\boldsymbol{b})$, then $\boldsymbol{a}>\boldsymbol{b}$. Moreover, if $i$ is non-null, then for all $\boldsymbol{a}$ and $\boldsymbol{b}$ in $\mathcal{Q}^{+}$that agree outside $i$, if $\boldsymbol{a}>\boldsymbol{b}$, then $L(\boldsymbol{a}) \tilde{\succ} L(\boldsymbol{b})$.

This condition ensures that your judgments about $\mathcal{K}$ and $\mathcal{Q}$, or the functions $v$ from (5.3) and $u$ from (5.1) and (5.2), are appropriately related. As a more technical assumption, we also need to assume that neither your axiologies nor your m-value judgments about $\mathcal{K}$ are uniform, or rank all prospects as equally (m-)good. So for

108 See Karni and Schmeidler $(1980,7)$. 
some binary relations $\geq_{i}$ on $\mathcal{O}$ and $\tilde{\Sigma}$ on $\mathcal{K}$ say that $\succeq_{i}$ is non-uniform if there are $a$ and $b$ in $\mathcal{O}$ such that $a>_{i} b$, and that $\check{\succeq}$ is non-uniform if there are $a$ and $b$ in $\mathcal{K}$ such that $a \tilde{\succ} 6$. We can now state the

Representation Theorem for Probabilities: Suppose that all your $\geq_{i}$ are $v N M$ conformable and non-uniform. If your $\succeq_{m}$ and your $\tilde{\succeq}_{m}$ are vNM-conformable and jointly satisfy the Consistency Axiom, if your $\succeq_{m}$ satisfies the Pareto Condition with respect to your $\succeq_{i}$, and your $\tilde{\Xi}_{m}$ is non-uniform, there's a unique probability distribution $P$ on $I$ and a function $u: I \times X \rightarrow \mathbb{R}$, unique up to positive affine transformation, such that for all $a$ and 6 in $\mathcal{K}$, all $\boldsymbol{a}$ and $\boldsymbol{b}$ in $\mathcal{Q}$, all $a$ and $b$ in $\mathcal{O}$ and all $i$ in $I$,

$$
\begin{gathered}
a \Sigma_{m} b \text { iff } \sum_{i \in I, x \in X} a(i, x) P(i) u(i, x) \geq \sum_{i \in I, x \in X} b(i, x) P(i) u(i, x), \\
\boldsymbol{a} \geq_{m} \boldsymbol{b} \text { iff } \sum_{i \in I, x \in X} \boldsymbol{a}(i, x) u(i, x) \geq \sum_{i \in I, x \in X} \boldsymbol{b}(i, x) u(i, x), \quad \text { and } \\
a \geq_{i} b \text { iff } \sum_{x \in X} a(x) u(i, x) \geq \sum_{x \in X} b(x) u(i, x),
\end{gathered}
$$

and such that if $i$ is null, $P(i)=0$, and if $i$ is non-null, $P(i)>0 .{ }^{109}$

This is a purely mathematical theorem. It says that if your $\succeq_{i}, \geq_{m}$ and $\tilde{\succeq}_{m}$ satisfy these conditions, there are mathematical functions that represent these relations. To turn it into a philosophically significant result, we must again add the relevant conceptual assumptions. So let me express more formally the judgmentexplication-or the idea that your credence in an axiology is the weight you give it in your m-value judgments. To restate the prospect-explication of intra-theoretic comparisons within the subjective framework we're now assuming, for your binary relation $\succeq_{i}$ on $\mathcal{O}$ and a function $u: X \rightarrow \mathbb{R}$, say that $u$ represents your $\geq_{i}$ ordinally if for all $a$ and $b$ in $\mathcal{O}$,

$$
a \geq_{i} b \text { iff } \sum_{x \in X} a(x) u(x) \geq \sum_{x \in X} b(x) u(x) .
$$

Furthermore, say that a function $u: X \rightarrow \mathbb{R}$ represents your $\succeq_{i}$ cardinally if for all $x, y, z$ and $t$ in $X$ and $n \in \mathbb{R}$, the value-difference between outcomes $x$ and $y$ is $n$ times as great as the value-difference between $z$ and $t$, according to your $T_{i}$, if and only if $(u(x)-u(y)) /(u(z)-u(t))=n$. The judgment-explication of intratheoretic comparisons says that if there is a function $u: X \rightarrow \mathbb{R}$, unique up to positive affine transformation, which represents your $\succeq_{i}$ ordinally, then $u$ represents your $\succeq_{i}$ cardinally. Now for binary relations $\succeq_{m}$ on $\mathcal{Q}$ and $\check{\succeq}_{m}$ on $\mathcal{K}$, a probability distribution

109 See the appendix (Section A.2) for a proof. 
$P$ on $I$ and a function $u: I \times X \rightarrow \mathbb{R}$, say that $(P, u)$ represents your $\succeq_{m}$ and $\tilde{\succeq}_{m}$ ordinally if for all $\boldsymbol{a}$ and $\boldsymbol{b}$ in $\mathcal{Q}$ and all $a$ and $b$ in $\mathcal{K}$,

$$
\begin{aligned}
& \boldsymbol{a} \succeq_{m} \boldsymbol{b} \text { iff } \sum_{i \in I, x \in X} \boldsymbol{a}(i, x) u(i, x) \geq \sum_{i \in I, x \in X} \boldsymbol{b}(i, x) u(i, x), \quad \text { and } \\
& a \widetilde{\succeq}_{m} b \text { iff } \sum_{i \in I, x \in X} a(i, x) P(i) u(i, x) \geq \sum_{i \in I, x \in X} b(i, x) P(i) u(i, x) .
\end{aligned}
$$

Furthermore, suppose that for some probability distribution $P$ on $I$ and some function $u: I \times X \rightarrow \mathbb{R}$, the crosscutting cardinal intertheoretic comparisons between all outcomes and all theories are the same as the ratios among the utility differences between these outcomes according to $u$, and that your credences in any axiology $T_{i}$ is $P(i)$-that is, for all $x, y, z, t$ in $X$, all $i, j, h, k$ in $I$ and $n \in \mathbb{R}$, the difference between the value of $x$, according to your $T_{i}$, and the value of $y$, according to your $T_{j}$, is $n$ times as great as the difference between the value of $z$, according to your $T_{h}$, and the value of $t$, according to your $T_{k}$, if and only if $(u(i, x)-u(j, y)) /(u(h, z)-u(k, t))=n$, with $p_{i}=P(i), p_{j}=P(j), p_{h}=P(h)$ and $p_{k}=P(k)$. I'll then say that $(P, u)$ represents your intertheoretic comparisons cardinally and your axiological probabilities quantitatively. The judgment-explication of your intertheoretic comparisons and axiological probabilities says that if there is such a pair $(P, u)$, with $P$ being unique and $u$ unique up to positive affine transformation, which represents your $\succeq_{m}$ and $\widetilde{\succeq}_{m}$ ordinally, and if for all $i$ in $I, u(i, \cdot)$ represents your $\succeq_{i}$ cardinally, then $(P, u)$ represents your intertheoretic comparisons cardinally and your axiological probabilities quantitatively. So we can interpret all utility functions $u(i, \cdot)$ jointly as value functions $G_{i}$, and the probabilities $P(i)$ as your credences $p_{i}$.

Given this explication, the following theorem holds:

Expected Value Theorem for Probabilities: Suppose that all your $\geq_{i}$ are vNMconformable and non-uniform. If your $\succeq_{m}$ and your $\tilde{\succeq}_{m}$ are vNM-conformable and jointly satisfy the Consistency Axiom, if your $\succeq_{m}$ satisfies the Pareto Condition with respect to your $\succeq_{i}$ and your $\tilde{\succeq}_{m}$ is non-uniform, then for all a and 6 in $\mathcal{K}$,

$$
a \tilde{\succeq}_{m} b \text { iff } \sum_{i \in I, x \in X} a(i, x) p_{i} G_{i}(x) \geq \sum_{i \in I, x \in X} b(i, x) p_{i} G_{i}(x) .{ }^{110}
$$

This is a non-mathematical result. But it's still only a conditional. It says that if your m-value judgments satisfy the relevant conditions, you're as a matter of fact

110 See the appendix (Section A.2) for the derivation of this theorem from the Representation Theorem for Probabilities and the judgment-explications. 
following (5.11)-and that is, EVM. Clearly, the converse is also true: if there exist a probability distribution $P$, and functions $G_{i}$ that represent your axiologies cardinally, and your judgments satisfy (5.11) (and its equivalent regarding $\mathcal{Q}$ ) with respect to them, then your judgments will satisfy the relevant conditions. So if we're interested in Subjectivist EVM, we can now turn this into an argument for the normative truth of (5.11). Denote the conditions in the antecedent of this theorem ('If your $\succeq_{m}$ and your $\tilde{\succeq}_{m} . .$. ') by ' $\mathcal{C}$ '. And say that you satisfy $\mathcal{C}$, or that your judgments are ' $\mathcal{C}$-conformable', if your judgments satisfy these conditions. If all your axiologies are vNM-conformable and non-uniform, we can then argue:

(R) your $\mathrm{m}$-value judgments are (subjectively) correct if and only if you satisfy $\mathcal{C}$;

(S) as a matter of logical equivalence, you satisfy $\mathcal{C}$ if and only if you follow Subjectivist EVM; therefore

(T) your m-value judgments are (subjectively) correct if and only if you follow Subjectivist EVM; Subjectivist EVM is true.

This is the main argument for a form of Subjectivist EVM. ${ }^{111}$

As mentioned, the Representation Theorem for Probabilities can be used to ground Objectivist EVM too. To that end, we can interpret the relations $\geq_{i}$ as denoting which prospects are better than which according to our axiologies-or as inducing the axiological orderings under consideration. We can interpret the relation $\succeq_{m}$ as denoting which prospects would be m-better than which for you, if axiological orderings had certain antecedently specified probabilities-or as inducing the intertheoretic comparisons that are reasonable for you to make between these orderings. And we can interpret the relation $\widetilde{\Xi}_{m}$ as denoting which prospects are $\mathrm{m}$-better than which, given your evidence-or as inducing the credences that your evidence warrants in the respective versions of our axiologies. The resulting argument would be similar to the argument outlined in Chapter 2: the m-value facts satisfy $\mathcal{C}$; if they satisfy $\mathcal{C}$, Objectivist EVM is true; therefore Objectivist EVM is true. For simplicity, I'll continue to focus on the subjectivist version of the theorem, the argument and EVM. But much of what I say applies mutatis mutandis to the objectivist version as well.

A brief note about the plausibility of the meta-axiological assumptions. Most of the conditions of the Representation Theorem for Probabilities are familiar from

111 Note that this argument is only sound if you make subjective judgments, about which prospects are m-better than which relative to your credences and the comparisons you make. If you make objectivist judgments, about which prospects are m-better than which relative to the credences your evidence warrants and the comparisons that are (constructively) correct, your judgments may satisfy $\mathcal{C}$ and still be false-by embodying unwarranted credences or mistaken comparisons. 
the Basic Representation Theorem. So my discussion of these conditions in Chapter 3 (and Chapter 6) clarifies how plausible they are as normative constraints on your (m-)value judgments. The problem of incommensurabilities carries over to the present context. Your $\succeq_{i}$ needn't satisfy Completeness, because according to some of your axiologies, some prospects might not be fully commensurable. And in addition, your $\succeq_{m}$ and $\widetilde{\succeq}_{m}$ needn't satisfy Completeness, because some of your axiologies may be less than fully commensurable to each other. However, in the context of your $\tilde{\succeq}_{m}$ on $\mathcal{K}$, there might be an additional reason for why your judgments needn't be complete. To assume that your $\tilde{\succeq}_{m}$ should satisfy Completeness is to assume that your credences ought to be sharp, or representable by single real numbers. If your credences can justifiably be 'fuzzy'-or less than fully sharpyour $\check{\beth}_{m}$ needn't be complete. And this is so even if all your axiologies are complete and fully commensurable. There are strong arguments to the effect that your non-normative credences needn't be precise, and these plausibly carry over to axiological credences. ${ }^{112}$ But since the problem of fuzzy credences is not specific to axiological uncertainty, I'll rule out fuzzy credences throughout this book. So this is another important restriction of our arguments. It's plausible that your axiological credences can justifiably be fuzzy, but I'll ignore this.

\subsection{Applying Subjectivist EVM in practice}

I'll presently discuss objections to the argument just outlined. But first, it might help to spell out how the theory of axiological uncertainty based on the Expected Value Theorem for Probabilities and the judgment-explication of credences can be applied in real-life cases. In principle, the theory is silent on this. EVM is a criterion of $\mathrm{m}$-betterness, not a procedure for how to arrive at m-value judgments in practice. So in principle we might satisfy EVM by just following the advice of a fortuneteller. But that seems unlikely. And taking our criterion of m-betterness as a decision procedure often seems a good way of arriving at m-value judgments. So let's see how this could be done. As an example, suppose you consider becoming a vegan. You believe that doing so would increase animal welfare by some amount, but reduce human welfare by $60 \%$ of that amount (since it would require resources you're currently using to your and other people's benefit). You're certain that welfare and only welfare has value, but uncertain about whether animal welfare is equally as valuable as human welfare. And you want to know whether it's $\mathrm{m}$-better for you to become a vegan. How do you answer this question?

112 See e. g. Joyce (2005; 2010); see e. g. Elga (2010) and White (2010) for arguments that subjective probabilities should be sharp. 
First, you need to determine what axiological orderings you're uncertain about. You need to determine your orderings $\geq_{i}$. To that end, you can consider simple examples like the following:

Tab. 5.1: Example to illustrate axiological orderings.

\begin{tabular}{lll}
\hline $\boldsymbol{a}$ & $\boldsymbol{b}_{\boldsymbol{q}}$ & \\
\cline { 2 - 3 } $\boldsymbol{p = \boldsymbol { q }}$ & Maria killed & status quo \\
\hline Bruin killed & & \\
\hline
\end{tabular}

If you choose prospect $a$, bear Bruin will be killed with certainty. If you choose prospect $b_{q}$, human Maria will be killed with probability $q$, and nothing will happen otherwise. Let's assume that their death would be equally bad for Bruin and Maria. Now if according to some axiology, $a \sim_{i} b_{1}$, the value of animal welfare is equally as great as the value of human welfare according to this axiology. If according to an axiology, $a \sim_{j} b_{0.5}$, the value of animal welfare is half as great as the value of human welfare according to it. Suppose you think that these two and only these two axiologies are plausible. Let's call them welfare-theory $\left(T_{W}\right)$, and species-weighted-welfare-theory $\left(T_{S W W}\right)$ respectively.

Next, you need to determine how your theories compare intertheoretically, or in what relative versions of these orderings you have credence. To that end, you have to consider your m-value judgments about $\mathcal{Q}$. This will be easiest with unnatural toy examples, like the following:

Tab. 5.2: Example to illustrate intertheoretic comparisons.

\begin{tabular}{llll}
\hline$a_{x}$ & & $b_{x}$ & \\
\cline { 1 - 2 } & $T_{W}$ & $T_{S W W}$ & $T_{S W W}$ \\
$\boldsymbol{p}_{W}=x$ & $p_{S W w}=(1-x)$ & $p_{W}=x$ & $p_{S W w}=(1-x)$ \\
\hline Maria killed & status quo & status quo & Maria killed \\
\hline
\end{tabular}

If you choose $\boldsymbol{a}_{x}$, Maria will be killed if $T_{W}$ is true, and nothing will happen if $T_{S W W}$ is true. If you choose $\boldsymbol{b}_{x}$, Maria will be killed if $T_{S W W}$ is true, and nothing will happen if $T_{W}$ is true. And these prospects are such that the probability of $T_{W}$ and $T_{S W W}$ are $x$ and $(1-x)$ respectively. So in judging that $\boldsymbol{a}_{2 / 3} \sim_{m} \boldsymbol{b}_{2 / 3}$, say, you'd judge that killing Maria was twice as bad on $T_{S W W}$ as on $T_{W}$. But suppose you actually judge that $\boldsymbol{a}_{1 / 2} \sim_{m} \boldsymbol{b}_{1 / 2}$. This means you think $\boldsymbol{a}_{x}$ and $\boldsymbol{b}_{x}$ are equally $\mathrm{m}$-good if $T_{W}$ and $T_{S W W}$ are equiprobable, or that the value of human welfare is 
the same on both theories. You might think this, say, because you think this is the simplest assumption, or the least radical way of reforming your credence in light of the evidence you've just found for $T_{W}$.

Finally, you need to determine what credence you have in these theories. To that end, you have to consider your $m$-value judgments about $\mathcal{K}$. This will again be easiest with unnatural toy examples, like the following:

Tab. 5.3: Example to illustrate axiological credences.

\begin{tabular}{llll}
\hline$a_{n}$ & & $\sigma_{m}$ & \\
\cline { 1 - 1 } & $T_{W}$ & $T_{W}$ & $T_{s w W}$ \\
\hline$n$ people killed & status quo & status quo & $m$ people killed \\
\hline
\end{tabular}

If you choose $a_{n}$ then $n$ people will be killed if $T_{W}$ is true, and nothing will happen if $T_{S W W}$ is true. If you choose $b_{m}$ then $m$ people will be killed if $T_{S W W}$ is true, and nothing will happen if $T_{W}$ is true. Their death would be equally bad for all of these people. So in judging that $a_{1} \approx_{m} b_{1}$, say, you'd judge it equally m-good to risk the death of a person conditional on $T_{W}$ as conditional on $T_{S W W}$. And since we already know you find these deaths equally bad on either theory, this means you'd assign both $T_{W}$ and $T_{S W W}$ the same probability. But suppose you actually judge that $a_{2} \tilde{\sim}_{m} b_{1}$. This means you think it's equally m-good to risk the death two people conditional on $T_{W}$ as the death of one person conditional on $T_{S W W}$. It means that the credence you have in $T_{W}$ is half of that you have in $T_{S W W}$.

So your judgments about these toy examples imply what axiological orderings you have credence in, how they compare, and how much credence you have in them. You can now use these probabilities and values to determine whether it's m-better for you to turn vegan. We were assuming that according to your nonnormative beliefs, your becoming a vegan increases animal welfare (a-welfare) by some amount $w$, but reduces human welfare (h-welfare) by $60 \%$ of that amount:

Tab. 5.4: Example to illustrate Subjectivist EVM.

\begin{tabular}{lllll}
\hline Becoming a vegan & & \multicolumn{2}{c}{ Not becoming a vegan } \\
\cline { 1 - 2 } & $T_{\boldsymbol{S} W w}$ & & $T_{\boldsymbol{W}}$ & $T_{\boldsymbol{S W W}}$ \\
\hline a-welfare $+w$, & a-welfare $+w$, & status quo & status quo \\
h-welfare $-0.6 w$ & h-welfare $-0.6 w$ & & \\
\hline
\end{tabular}


We can assume (without loss of generality) that the value of the status quo is 0 on both theories, and that the disvalue of decreasing human welfare by $w$ is -1 . Note that according to the theory you find more plausible $\left(T_{S W W}\right)$, it's better not to become a vegan. The value of becoming a vegan, according to $T_{S W W}$ is

$$
\frac{1}{2}-\frac{6}{10}=-\frac{1}{10}<0 .
$$

Nonetheless, it's m-better for you to become a vegan. The expected value of becoming a vegan is

$$
\frac{1}{3}\left(1-\frac{6}{10}\right)+\frac{2}{3}\left(\frac{1}{2}-\frac{6}{10}\right)=\frac{1}{15}>0 .
$$

So relative to your credences, it's m-better for you to become a vegan, even though you think it's more likely worse.

This is at least the beginning of the full story of how our theory can-in Savage's terms-help you to 'police [your] own decisions for consistency and, when possible, to make complicated decisions depend on simpler ones' $(1954,20)$. Of course, in practice, you might also have credence in other versions of these theories which compare differently; or you might have credence in other speciesweighted-welfare-theories, which imply a different ordering; or you might have still other views that affect this decision. Then the above calculations are only a first approximation of whether it's m-better for you to become a vegan. Your calculation will be more accurate the more of these alternatives you take into account. Moreover, you might also have an intuition concerning the $\mathrm{m}$-value of becoming a vegan. If you intuitively judge it $m$-worse to turn vegan, your m-value judgments can't all be correct. So you need to revise some of these judgments, until you attain a reflective equilibrium in which they satisfy all of our constraints. As an aside, note that the ability to construct unnatural prospects like $\boldsymbol{a}_{x}, \boldsymbol{b}_{x}, a_{n}$ and $b_{m}$ is helpful in thinking about these questions. In this respect, as indicated on page 24 , the unnatural aspects of our framework are actually an advantage.

\subsection{Objections and implications}

The argument in Section 5.1 again suggested that representation theorems can serve the two foundational purposes indicated in Section 1.2: clarify what EVM means, and corroborate that it is true. But especially with respect to the notion of credences, many philosophers are sceptical of the significance of representation theorems for these purposes. Christopher Meacham and Jonathan Weisberg, for 
instance, claim that 'representation theorems cannot serve either of these foundational purposes', and that, 'we should [...] lay the foundations of decision theory on firmer ground' than that provided by such theorems $(2011,641)$. So it's time to address some objections. Most of these objections have been levelled against preference-based representation theorems in decision theory. But they apply $m u$ tatis mutandis to Subjectivist EVM in the context of axiological uncertainty. And I'll only be concerned with that. I'll argue that the judgment-explication is the best explication of axiological credences. But I won't claim that preference-based theorems can serve these purposes in decision theory or epistemology more generally. That's another question. ${ }^{113}$

\section{Relationship to the ordinary concept}

A technical concept may diverge from the ordinary language notion in some respects. It may be more precise, and may perhaps also shift the original meaning slightly. However, if it's to be an explication of that notion-rather than a stipulative definition of a completely new term-it shouldn't deviate too much from the explicandum. Preference-based definitions of credences have been criticised in this respect, and some worries carry over to our context. Here's a first worry. Intuitively, most of us have varying credences in axiologies: we find some axiologies quite plausible, others somewhat less, and still others highly unlikely. Yet we generally don't satisfy $\mathcal{C}$. So we can rarely be ascribed credences as defined by our explication. Thus our technical notion diverges from its explicandum. Among others, Meacham and Weisberg (2011) raised this objection against preference-based definitions in decision theory. ${ }^{114}$ Referring to the technical notions of utilities and degrees of belief as 'utilities ${ }^{\star}$ ' and 'degrees of belief*', they say:

To make degrees of belief* and utilities ${ }^{\star}$ relevant to epistemology and normative decision theory, these states must be linked to the states that are the topic of our normative theorizing in these domains. And since agents like us generally don’t have degrees of belief ${ }^{\star}$ and utilities $^{\star}$, it’s hard to see how they're relevant. $(2011,655)$

The same worry applies mutatis mutandis in our context.

113 In particular, I'll ignore the debate about probabilism -the view that your credences should satisfy the axioms of probability theory. According to the judgment-explication and my main argument, probabilism is true. But I don't understand it as an argument for probabilism. I understand it as an argument for how your credences and values should interact to determine your m-value judgments.

114 Eriksson and Hájek (2007, 200 f.; 203 f.) and Zynda (2000, 62) make the same point. 
There are three replies to this objection. First, we must keep the purpose of our explication in mind. Since you'll rarely satisfy $\mathcal{C}$, the specific explication I've given may be relatively useless in descriptive or predictive accounts of your mental states or behaviour. But Subjectivist EVM isn't a descriptive or predictive account. It's a normative account of what your m-value judgments should be, or of when they're correct. It says your m-value judgments are (subjectively) correct if and only if you satisfy $\mathcal{C}$ in one way or another; and that if you do, you satisfy EVM with respect to your credences. The fact that we can't assign credences to you on the basis of our explication if you fail to satisfy $\mathcal{C}$ is irrelevant for whether this claim is convincing, or close enough to our ordinary concept of credences. All that's relevant is that our explication resembles our intuitive concept when you satisfy $\mathcal{C}$. And Meacham and Weisberg's argument does nothing to challenge that. Against the specific normative interpretation of EVM I've adopted, their argument is simply a red herring.

Second, we need to distinguish between the specific explication I've given and the general idea behind it. The general idea of the judgment-explication is simply that your credence in an axiology is the weight you give it in your m-value judgments. And this idea isn't limited to the ideal cases where you satisfy $\mathcal{C}$, or to the narrowly normative interpretation of EVM I've invoked. The Representation Theorem for Probabilities shows that if you satisfy $\mathcal{C}$, there's a unique weight you give all your axiologies. But even when you don't satisfy these conditions, there might be facts about the weights you give your axiologies in your m-value judgments. And through slightly broader interpretations of our general explicative idea, and perhaps slightly more complex theorems, these facts might still allow credence-interpretations of you. For instance, suppose you don't satisfy Completeness. Intuitively, this might mean you don't assign unique probabilities to your axiologies in the manner my explication presupposes, but have fuzzy credences in them. And our general explicative idea could capture this. There are representation theorems without the Completeness axiom, which in such cases can represent you as assigning a range of weights to your axiologies. Thus we could interpret you as assigning probability-ranges to them. Alternatively, suppose you don't satisfy $\mathcal{C}$ for the full range of axiologies $\mathcal{T}$ (and the corresponding sets $\mathcal{K}$ and $\mathcal{Q}$ ). You satisfy $\mathcal{C}$ only for the set $\mathcal{T} \backslash T_{i}$ (and the corresponding prospects). Intuitively, this might mean you've decided how likely all other axiologies are, relative to each other. But you haven't yet made up your mind about what credence you assign to $T_{i}$. Again, our general idea could capture this. We could use the Representation Theorem for Probabilities, and my judgment-explication, as applied to the set $\mathcal{T} \backslash T_{i}$-and add the caveat that you haven't yet fully made up your mind regarding $T_{i}$. Or again, suppose you don't satisfy $\mathcal{C}$ for the full set of outcomes $X$ (and the corresponding sets $\mathcal{K}$ and $\mathcal{Q}$ ). You satisfy $\mathcal{C}$ only for the set $X \backslash x$ (and the 
corresponding prospects). Intuitively, this might mean you've basically decided how likely your axiologies are. But for some reason, you just get confused when considering the outcome $x$. Again, our general idea could capture this. We could use the Representation Theorem for Probabilities, and my judgment-explication, as applied to the set $X \backslash x$-and add the caveat that $x$ seems to confuse you. Or perhaps all these qualifications apply: you satisfy only Transitivity, Independence and Continuity, with respect to a restricted set of axiologies, and a restricted set of outcomes. Even then, there might be certain facts about the weights you give your axiologies, which would allow certain claims about your credences in light of our explication.

Third, we must not be too lenient in assigning credences to people. The cases I've just described are still to some extent ideal: they presuppose that you satisfy Transitivity, Independence and Continuity with respect to some theories and outcomes. Perhaps when people don't even show any such traces of satisfying $\mathcal{C}$, there's no way for our explication to assign credences to them. But this seems intuitively plausible too. Intuitively, some people simply don't allow any meaningful assignments of degrees of belief. They're just too incoherent. So arguably, these remaining limitations even speak in favour of our explication. The core idea of the judgment-explication can explain when credence-assignments intuitively become impossible.

A more promising strategy might be to attack the core idea of the judgmentexplication directly, and question whether the explication is adequate when you satisfy $\mathcal{C}$. And this may be questioned. One pertinent worry is that our ordinary notion of credences is much richer than the concept I've introduced. Among others, David Christensen (2001) stressed this point in decision theory-suggesting that 'the preference-based definition leaves out important parts of our pretheoretic notion' $(2001,361) .{ }^{115}$ For one thing, 'a person's beliefs [...] affect the way she behaves in countless ways that have nothing directly to do with the decision theorist's paradigm of cost-benefit calculation' (2001, 361). For another thing, degrees of belief not only help to explain behaviour, but also 'other psychological states and processes' (2001, 361). Your self-deprecating beliefs may explain why you're performing poorly in a competition, or why you're being sad or afraid, or why you release stress hormones or are physically unhealthy. Beliefs are involved in a plethora of explanatory connections, even when our preferences satisfy the

115 The same point is endorsed by Eriksson and Hájek (2007, 208). Meacham and Weisberg (2011, 646) also highlight the rich explanatory connections of beliefs (though not as an objection against what they call the 'Explicative View', and what is basically the view I suggested); see also Hájek (2008, 803 ff.). 
axioms. This being so, Christensen claimed, 'the move of settling on just one of these connections-even an important one-as definitional comes to look highly suspicious' (2001, 362). And this worry carries over to our context. Your axiological beliefs arguably play a much richer role than just determining your m-value judgments: they may explain your behaviour, your immediate reactions, attitudes and emotions and so on, even when you satisfy $\mathcal{C}$.

This is a more pertinent worry. But again it can be answered. Christensen focused on Patrick Maher's (1993) understanding of probabilities and utilities, according to which 'an attribution of probabilities and utilities is correct just in case it's part of an overall interpretation of the person's preferences that makes sufficiently good sense of them and better sense than any competing interpretation does' (1993, 9). Christensen objected:

a given interpretation of an agent's degrees of belief might maximize expected-utility fit with the agent's preferences while a different interpretation might fit much better with other psychological-explanatory principles. In such cases of conflict [...] there's no guarantee that the best interpretation will be the one on which the agent's preferences accord perfectly with maximizing [expected utility]. $(2001,362)$

But my understanding of the explication differs from Maher's. I don't offer it as the best overall interpretation of an agent. On the contrary, at least if 'best' means 'descriptively best' as opposed to 'best for our purposes', I'd doubt there generally is a uniquely best interpretation. Suppose in my m-value judgments I give much more weight to the welfare- than the human-welfare-theory. But in my emotional reactions, implicit attitudes, hormone levels and in my actual behaviour, I give somewhat more weight to the human-welfare- than to the welfare-theory. In which theory do I then have a higher degree of belief? Do my reactions and behaviour show that I don't really have a higher credence in the welfare-theory? Or do I act and react against my true credences, due to akrasia or biases or whatever? Very plausibly, our intuitive concept of credence isn't sharp enough to imply anything definitive in all such cases. There is often no uniquely 'best interpretation'. There are several viable interpretations that don't seem outright misleading, and that might all pick out a theoretically useful phenomenon. ${ }^{116}$ All I claim is that the judgment-explication is one of them. If you satisfy $\mathcal{C}$, and thus give each axiology a constant weight in your m-value judgments under uncertainty, it's not

116 There's a literature on whether, if you profess to believe that $p$ but act contrary to that professed belief, you truly believe that $p$ (see e. g. Schwitzgebel 2010 for an overview, and a position similar to the one expressed above). I'm not aware of a discussion of such cases with regards to a graded notion of belief. 
determinately wrong or completely misleading to call the relevant weights your 'credences'. And it picks out something that's useful for our purposes.

Let me elaborate on the latter point. Why focus on your m-value judgments, of all possible connections, to explicate credences? There are at least two reasons. First, we want to say you should satisfy EVM with respect to your 'credences'. So we need to pick out 'credences' that should be relevant in your decisionmaking. But many aspects Christensen emphasises arguably shouldn't be. Many of us won't have the moral emotions we judge to be fitting. ${ }^{117}$ Our implicit attitudes often contradict what we explicitly claim to believe and take to reflect our evidence-and often in a sexist or racist manner. ${ }^{118}$ And presumably, many of us fail to act in accordance with what we judge to be good. ${ }^{119}$ And this isn't surprising. Among other things, our judgments can adapt to new evidence very quickly. Our behaviour, emotions and implicit attitudes are much more resistant to such changes. But plausibly, when there's a conflict between your considered judgments and other aspects of your psychology, you shouldn't satisfy EVM with respect to the latter. You should satisfy EVM with respect to the 'credences' induced by your judgments. Our explication picks out something relevant to what you ought to do.

Second, our explication is very simple. In response to Christensen's conflict cases, we could provide an explication involving other aspects of our psychology. For example, we could introduce conditions on attitudes, of the sort: 'if you have a favouring attitude for $a$ over 6 , then for any $c$ in $\mathcal{K}$ and any $p \in] 0,1$, you have a favouring attitude for $p a+(1-p) c$ over $p b+(1-p) c$ '. These conditions may imply an attitude-relative 'credence'-function. Perhaps we could do the same for your behaviour, your hormone level, and so on. And we could then take a weighted average of these relativized 'credence'-functions to get your overall credence-function. Or we could assume bridging principles of the form 'if you judge that $a$ is m-better than $b$, you have a favouring attitude for $a$ over $b$ ', guaranteeing that all these credence functions are the same. No one has done this, and it would involve serious difficulties. ${ }^{120}$ But even if it were possible, the complexity of such an account of

117 See e. g. Greene et al. $(2001,2107)$ on people judging 'in spite of their emotions'.

118 See e. g. Greenwald and Banaji (1995), Gaertner and McLaughlin (1983) or Dovidio and Gaertner (2000) for relatively early works; Strohminger et al. (2014) for a recent methodological survey about implicit moral attitudes.

119 See e. g. the findings of Schwitzgebel and Rust (2014) or Schwitzgebel (2014), suggesting that more stringent moral views don't imply more stringent moral behaviour.

120 For instance, different people seem to react differently to the belief that one prospect is $\mathrm{m}$ better than another; no such reaction might be a necessary condition for that belief, and the choice of any one might be somewhat arbitrary. 
credences would be a major drawback. Our explication is much simpler. So the judgment-explication may not provide the uniquely overall best interpretation of you. There often won't be any such interpretation. But our explication picks out a feature of 'beliefs' that's descriptively very salient, normatively relevant, and still comparatively straightforward.

Here's a third, related worry. One might worry, not that there are additional connections between our credences and our psychology, but that sometimes the presumed connection with our m-value judgments doesn't exist. Lina Eriksson and Alan Hájek (2007) have claimed this with respect to standard preferenceexplications in decision theory:

Credences and preferences are certainly separable in thought, and sometimes in practice. Imagine a Zen Buddhist monk who has credences but no preferences [i. e., 'is indifferent among all things']. [...] If the monk is conceptually possible, then any account that conceptually ties credences to preferences is refuted. [...] Or consider a chronic apathetic who has lost all his desires, but who has kept all his credences. To be sure, these characters are not recognizably like us, although some of us may approximate them over certain domains, and to the extent that we do, bets and preferences more generally ill-reflect our true credences. $(2007,194)$

Others have provided similar arguments. ${ }^{121}$ And a similar point seems to apply in our context. Axiological credences and m-value judgments are 'separable in thought', it seems, since we can imagine someone who has axiological beliefs but doesn't make any m-value judgments.

But what precisely is the argument here? On the face of it, first, mere 'approximation' of monk-like apathy doesn't distort preference-based explications at all. It will simply result in an attenuated utility function, and this seems precisely appropriate. Second, the perfectly indifferent monk is such that our theorem just doesn't apply to them. The Expected Value Theorem for Probabilities presupposes that you make (non-uniform) m-value judgments. Thus monk-cases can't show that the explication is flawed when it applies. ${ }^{122}$

But perhaps Eriksson and Hájek raise a deeper worry. As applied to our context, their argument seems to be that the mere possibility of axiological credences

121 See Christensen $(2001,363)$.

122 True, there's arguably a difference between the monk and someone with, say, intransitive preferences: the latter is (presumably) making some sort of mistake, whereas the monk perhaps needn't make any mistake. So the monk shows that the Representation Theorem for Probabilities can't ground a fully general normative theory. But I've already admitted that many constraints in that theorem are too strong as normative constraints, and are only plausible given certain restrictions or simplifications (e. g., the assumption that all axiologies are non-uniform). 
and m-value judgments coming apart shows that any definition of the former in terms of the latter must be flawed. If they can come apart, the thought seems to go, any connection between them must be contingent and can't be conceptual. But that's simply a non-sequitur. A condition may be sufficient but not necessary for a concept to apply, and this may be a conceptual truth. It seems to be a conceptual matter, say, that if you have the ability repeatedly to play the first prelude from the Well-Tempered Clavier, then you know how to play it. And yet, you can also know how to play it without having that ability, as when your arms are broken or all pianos have been destroyed. Our explication only says that if you satisfy $\mathcal{C}$, then as a matter of explication, you have the credences that make you satisfy EVM. And this may be correct, or a legitimate explication, even if intuitively you may have credences even if you don't make m-value judgments.

Perhaps Eriksson and Hájek have in mind yet another argument: that we anyway need an explication of the perfect monk's credences; that that explication can't be preference-based; that it will apply to any other agent as well; and that therefore the preference-explication is redundant. However, for reasons I've indicated, it's unclear whether we can give an adequate explication of someone's quantitative credences if they don't make any m-value judgments. So unless Eriksson and Hájek present a better or more general explication (which they don't) this argument doesn't get off the ground. And more generally, perfectly indifferent monks are very rare, or indeed presumably inexistent. And so are people who don't make any m-value judgments. If we have an otherwise useful explication, that's silent on these highly exceptional cases, that seems like a limitation we can live with. ${ }^{123}$

\section{Normative relevance}

Let's turn to a different set of worries about the judgment-explication. These worries concern the normative relevance of the resulting theory. They challenge

123 There are other objections against preference-based explications in decision theory; Eriksson and Hájek (2007) offer an overview. Another worry that carries over to our context is that explicating credences in terms of preferences gets the order of explanation wrong (see Eriksson and Hájek 2007, 207 f.). I've replied to the equivalent objection concerning value in Section 3.3. A similar reply could be given concerning credences. As far as I see, the remaining objections don't apply to my explication of axiological credences. In particular, a prominent objection against explicating degrees of belief in terms of preferences is that this explication is overly 'pragmatic', reducing a doxastic attitude (credence) to a conative one (preferences) (see Joyce 1999, $89 \mathrm{ff}$. or Eriksson and Hájek 2007, 194). However, my explication explains a doxastic attitude (axiological credences) in terms of another doxastic attitude (m-value judgments). So this objection doesn't apply. 
that Subjectivist EVM as understood by the judgment-explication can play the normative role we want a theory of axiological uncertainty to play. A first such worry is that Subjectivist EVM is normatively trivial. A theory of axiological uncertainty should constrain your judgments about what's m-best. But Subjectivist EVM seems to take your m-value judgments as given to define your credences and values. So it may seem that it will just guarantee that your $\mathrm{m}$-value judgments are correct, and thus can't constrain or guide you at all. ${ }^{124}$

That's a misunderstanding. Subjectivist EVM does put constraints on you. These constraints are simply the axioms. In the version I've outlined, your mvalue judgments can't be correct if you violate $\mathcal{C}$. So you may use these conditions to guide your evaluation of prospects, or make more complex evaluations depend on simpler ones. What's true is that-as far as the correctness of your m-value judgments as subjective m-value judgments is concerned-these conditions are all that Subjectivist EVM implies. And these conditions only rule out certain sets of m-value judgments, but no individual judgment in itself: they're mere coherence constraints (as I've put it), rather than unconditional local constraints on individual judgments themselves. Thus Subjectivist EVM reduces to a set of coherence constraints. This doesn't mean that any $\mathcal{C}$-conformable set of judgments is as good as any other in all respects. Some such judgments will reflect inadequate credences, and thus be epistemically problematic, say. But on Subjectivist EVM, your m-value judgments are correct as subjective m-value judgments relative to your credences if and only if they're $\mathcal{C}$-conformable.

Here's a second, related worry. Even if that's not outright trivial, one might still find it disappointing. Meacham and Weisberg do. They argue that the view that you ought to maximise the expectation of utility ${ }^{\star}$ relative to your credences ${ }^{\star}$ (in the technical senses of these terms) is 'prescriptively useless' $(2011,656)$ or 'uninteresting' (2011, 642; 645; 655; 661): 'Normative decision theory applies only to agents who have degrees of belief and utilities. But agents who have degrees of belief $^{\star}$ and utilities ${ }^{\star}$ are automatically [...] expected utility maximizers with respect to them.' So, they suggested, 'it will be true by definition that all agents subject to the norms of decision theory satisfy them'. $(2011,653)$ The worry is that the norm to maximise expected utility only applies to you if you have credences and utilities; but that once you have credences ${ }^{\star}$ and utilities ${ }^{\star}$ you automatically maximise

124 Sepielli $(2010,169)$ raised this objection against preference-based explications in decision theory: 'The standard way of assigning credences and utilities in decision theory assigns them in such a way that the agent's preferences will necessarily come out as maximizing expected utility. Since the going assumption in decision theory is that maximizing expected utility is necessarily rational, this means that agents will necessarily have fully rational preferences.' This is 'highly counterintuitive' $(2010,168)$, he says. 
expected utility with respect to them. But that's again just false. The coherence conditions $\mathcal{C}$ of Subjectivist EVM don't just apply to you once you have axiological credences. They apply to you whenever. They say you always ought to make $\mathrm{m}$-value judgments that satisfy Completeness, Transitivity, Independence, and so on. We might say Subjectivist EVM requires you, among else, to have axiological credences.

Meacham and Weisberg consider this reply in a footnote. But all they say in response is that

this proposal represents a substantive shift in the content of normative decision theory. We're no longer dealing with the same norms, and these replacements can't do the same work as the originals. For example, normative decision theory is supposed to say which of an agent's options she ought to take. But the injunction to have degrees of belief and utilities will be silent on this, since every option will maximize expected utility relative to some pair of probability and utility functions. (2011, 648, n.15)

This is merely to say that if we thought EVM could imply local constraints, Subjectivist EVM constitutes a shift in our understanding. But it doesn't show why that should make it uninteresting, or why we thus have to 'lay the foundations of decision theory on firmer ground' than that provided by representation theorems (2011, 641).

In fact, the shift from an understanding of EVM on which it implies local to one on which it implies coherence constraints is much less dramatic than it may seem. On the one hand, at least on a reflective-equilibrium-type view in (meta-)axiological epistemology, there's anyway ultimately just coherence constraints. Suppose we can take axiological credences as primitives, and have some intuitive, introspective sense of what our credences are. If the numbers you gathered through introspection imply m-value judgments you find highly implausible (giving far too much weight to the average-welfare-theory, say) you shouldn't stick slavishly to these judgments. You should adjust your 'credences' until they cohere with a set of m-value judgments you find plausible. Thus ultimately, our norms concerning m-value judgments anyway reduce to coherence constraints. In Subjectivist EVM this reduction is internal to the theory. But it's not clear why that should be problematic. ${ }^{125}$ On the other hand, nothing in the judgment-explication implies that you may not also, say, consult your feelings of confidence when determining a set of m-value judgments. If you start with an intuitive list of numbers, and these

125 In fact, at least in the present case, I think it's preferable to have the coherence constraints built into the theory. Note that according to EVM as understood in terms of the Simple Explication, your first (extremely implausible seeming) $\mathrm{m}$-value judgments were true as subjective m-value judgments. That seems to show that, in itself, this theory is of little normative significance. 
numbers lead you to a set of m-value judgments that satisfy our conditions and that you find plausible, there's nothing wrong with that. The reduction to coherence constraints doesn't prohibit the use of introspection or local constraints in practice.

Here's a third worry Subjectivist EVM invites. If it normatively boils down to $\mathcal{C}$, why should it even matter whether we interpret your m-value judgments this way or another once you satisfy $\mathcal{C}$ ? It now seems all the work is done by the axioms, and that the formula (5.11) for EVM has become entirely redundant. So why should we even care about the EVM-representation? Why should we care about the relevant theorem and the bulk of my argument in this chapter?

The reason is that we may ultimately not just be interested in the truth of your $\mathrm{m}$-value judgments as subjective m-value judgments. As far as that is concerned, the formula for EVM indeed doesn't add an additional norm. But we may also be interested in whether your m-value judgments reflect reasonable credences. And the EVM-representation helps express the connection between the theory of axiological uncertainty on the one hand, and axiological epistemology on the other. Judging that it's m-better to benefit people significantly more than non-human animals, say, seems epistemically unreasonable. But it's not perspicuous from the axioms alone how we can capture this in a unified way. Representation theorems show that if your judgments satisfy $\mathcal{C}$, you attach a constant weight to each axiology. So they allow us to say, not just that this or that m-value judgment is unreasonable, but that you're giving too much weight to speciesism. And if we can interpret these weights as credences, that's a congenial way to link the norms of the theory of axiological uncertainty to epistemology. It does justice to the intuitive idea that some axiologies are more plausible or likely true than others, and that these likelihoods are relevant in determining $\mathrm{m}$-value. And it allows us to say that the normative relationship between our evidence and these weights is one between evidence and a doxastic state, which may provide more unity to our overall picture of what an epistemic norm is, or what norms there exist. ${ }^{126}$

There's a fourth, more specific worry about employing coherence constraints on your m-value judgments. We all have some pretheoretic preferences, and can thus use coherence constraints to determine what further preferences we ought to have in cases where we haven't yet formed any. So coherence constraints might be good and well in standard decision theory. But one might worry that we can't make any $\mathrm{m}$-value judgments without prior theoretic guidance. Indeed this, one

126 Representation theorems can also serve a practical purpose. Instead of working with the axioms alone, it's often easier to derive your probabilities and values from simple cases, and then apply the formula for EVM for more complex ones. That's what I did in Section 5.2. 
might say, is why we need a theory of axiological uncertainty in the first place. Hence such a theory must be able to constrain us without assuming that we make prior m-value judgments, on pain of presupposing that we know already what it was supposed to tell us. This was argued by Brian Hedden, who reports to have 'few if any brute intuitions' $(2016,114)$ concerning which prospects are m-better than which, and suspects that this is true for most people. ${ }^{127}$

This is a more pertinent worry. But I'm much less pessimistic than Hedden about our ability to make m-value judgments. Axiological uncertainty raises difficult philosophical questions and presents intricate formal problems. But in an important sense, it's also a fairly straightforward phenomenon. It can be explained to non-specialists in a couple of sentences. And it doesn't seem to engender anything like intellectual paralysis as far as m-value judgments go. I suspect most people would say they aren't entirely certain about the value of equality or deserved punishment or the creation of an extra person. But I also suspect that, when pressed to judge in light of this uncertainty, most people would consider it m-better to bring about an equal distribution of some amount of welfare rather than a grossly unequal distribution of a marginally greater amount of it; or mbetter to punish the guilty rather than an innocent person for some moral wrong, even if this involves somewhat greater costs for society; but perhaps m-worse to create a person and thereby greatly harm an existing one rather than to leave the population as it is. Or perhaps they'd judge differently. But I'd be surprised if people were entirely clueless about which of such prospects are m-better. If anything, a sense of cluelessness seems to be post-theoretic, issuing perhaps from one's view about the problem of intertheoretic comparisons or one's metaethical assumptions.

Now of course, there will be many cases where we lack a firm intuition. We won't have a firm intuition about precisely which increase in overall welfare makes an unfair distribution equally as m-good as a fair one. But this needn't indicate a defect. Recall what I said in the last chapter. Plausibly, your m-value judgments needn't satisfy Completeness: there often aren't any positive facts about which

127 This worry is analogous to one about Harsanyi's theorem. Standardly, the theory of 'social preferences' is supposed to take individual preferences as inputs and tell you which of your prospects is socially best. But Harsanyi's theorem starts with social and individual preferences, and then tells you that if they satisfy certain axioms, you can pick utility functions that represent them and relate in a certain way. Again, Harsanyi's axioms might well help you derive some social preferences if you know some such preferences to start from. But you might worry that you don't have brute intuitions about what's socially best and thus can't know any such preferences-in particular, not unless you're antecedently told how to make interpersonal comparisons of welfare (see Hedden 2016, 114). 
prospects are m-better than which, since our axiologies aren't fully commensurable. And if this is so, our lack of firm intuitions needn't indicate that there are facts we're not grasping, and that our intuitions are poor. It may indicate that there aren't any facts, and that our intuitions are a good guide to that. The absence of a firm intuition might be the intuition of the absence of a fact.

Even if this is granted, and the practical usefulness of our theory accepted, there might be a fifth and final worry. One might object that a reduction to coherence constraints just makes EVM too permissive-that it's simply wrong that any judgments satisfying $\mathcal{C}$ are correct.

But there's an answer to this too, and it's perhaps a general answer to all remaining worries concerning the normativity of Subjectivist EVM. Recall that according to Subjectivist EVM, such judgments are only correct as subjective mvalue judgments. They might of course still reflect unreasonable credence distributions over axiological orderings, or implicate credences in versions of these orderings, or intertheoretic comparisons, that violate our prior constructivist norms. In other words, your judgments might still be false by the lights of Objectivist EVM. Subjectivist EVM only says they're correct by subjectivist standards. And any theory of subjective m-value is necessarily rather permissive. On any such theory, your m-best prospect depends on your credences. Hence no such theory will imply a local m-value judgment unless something about your credences is known. And it will render correct whatever judgments are implied by your credences. The difference is merely that on Subjectivist EVM, your credences are determined by your m-value judgments, rather than your brute introspection, say. But as I've argued, that doesn't seem problematic.

I've granted that there are reasons for thinking objective m-value is ultimately more important normatively speaking, or more more naturally the object of our judgments. But that doesn't threaten Subjectivist EVM as a theory about subjective m-value. And more importantly, it doesn't threaten the general approach of this book or this chapter. If you think objective m-value is more important, you can understand the Expected Value Theorem for Probabilities, and the argument it grounds, in objectivist terms-as indicated on page 94. It's not true that any judgments about objective m-value satisfying $\mathcal{C}$ will be correct. You might make $\mathcal{C}$-conformable judgments that imply unwarranted credences. So Objectivist EVM doesn't boil down to coherence constraints. And any remaining worries one might have about such constraints might be answerable by focusing on it, rather than its subjectivist cousin. 


\subsection{Conclusion: Weighted Value Maximisation}

Let me conclude. In this chapter, I've examined the question of how to understand axiological credences. I argued that we shouldn't take such credences as primitives, and introduced the judgment-explication to explain them. According to this explication, your credence in an axiology is the weight you give this axiology in your m-value judgments. After providing the formal background for this explication, I argued that it's adequately related to our intuitive concept and picks out something normatively relevant-or doesn't trivialise EVM, make it uninteresting or redundant. This suggests an explication of credences in terms of representation theorems on your m-value judgments is the best explication we can get.

More fundamentally, I've argued that it's preferable to interpret the weights that axiological orderings have in determining $\mathrm{m}$-value as being composed of their value function on the one hand, and their probability on the other. If we can make this distinction, we can capture the idea that some axiologies are more likely than others, and that these likelihoods are relevant in determining m-value. And we can thus express the intuitive connection between the theory of axiological uncertainty and axiological epistemology. However, I've admitted that in light of the import of the axioms, the distinction might be somewhat less relevant than it first appeared. So it might be worth indicating how the alternative view, which eschews the distinction between probabilities and values altogether, can be axiomatised in our framework. We can do that with a simple analogue of the Expected Value Theorem, concerning $\mathcal{K}$ instead of $\mathcal{Q}$. Define for each $i$ in $I$ a function $K_{i}: \mathcal{K} \rightarrow \mathcal{O}$; $a \mapsto K_{i}(a)$, such that for all $x$ in $X$,

$$
K_{i}(a)(x)=a(i, x) .
$$

The mapping $K_{i}$ thus formally turns a prospect $a$ in $\mathcal{K}$ into the prospect that $a$ represents, given $T_{i}$. Now for a set of binary relations $\left\{\geq_{i} \mid i \in I\right\}$ on $\mathcal{O}$ and a binary relation $\tilde{\underline{z}}$ on $\mathcal{K}$, define the

Pareto Condition for K: If for some $a$ and $b$ in $\mathcal{K}, K_{i}(a) \sim_{i} K_{i}(b)$ for all $i$ in $I$, then $a \approx b$; and if $K_{i}(a) \geq_{i} K_{i}(b)$ for all $i$ in $I$, and $K_{j}(a) \succ_{j} K_{j}(b)$ for some $j$ in $I$, then $a \tilde{\succ} 6$.

That your $\widetilde{\beth}_{m}$ should satisfy this condition is only plausible if you don't rule out the truth of any axiology under consideration completely. If there's some $T_{j}$, which you believe with certainty is false, the second clause of the condition is implausible with regards to that theory. So to ground an argument on this condition, we need to restrict the set of theories under consideration to those you don't rule out completely. The simplest way to do so is to restrict the theories to those for which 
your $\widetilde{\Xi}_{m}$ does, as a matter of fact, satisfy the Pareto Condition for $\mathcal{K}$. In any case, the following theorem holds:

Weighted Value Theorem: Suppose that all your $\geq_{i}$ are $v N M$-conformable, and that your $\tilde{\geq}_{m}$ satisfies the Pareto Condition for $\mathcal{K}$ with respect to them. If your $\widetilde{\Xi}_{m}$ is $v N M$-conformable, there's a function $u: I \times X \rightarrow \mathbb{R}$, unique up to positive unitcomparable transformation, that represents each axiology cardinally, and is such that for all $a$ and $b$ in $\mathcal{K}$,

$$
a \widetilde{\Sigma}_{m} b \text { iff } \sum_{i \in I, x \in X} a(i, x) u(i, x) \geq \sum_{i \in I, x \in X} b(i, x) u(i, x) .{ }^{128}
$$

In other words, if your m-value judgments satisfy the von Neumann-Morgenstern axioms and the Pareto Condition for $\mathcal{K}$, then to each axiology you're attaching a constant weight in the form of a utility function. This doesn't establish that we can treat such a function $u(i, \cdot)$ as a product $p_{i} G_{i}$ of your credence in, and the value function of, theory $T_{i}$. So (5.15) expresses Weighted Value Maximisation, rather than EVM. This is a genuine theory of axiological uncertainty. To my knowledge, no one has defended that theory so far. If we can establish EVM, EVM is preferable to Weighted Value Maximisation. But Weighted Value Maximisation is a relevant alternative.

128 See the appendix (Section A.2) for a proof. 